\title{
revisión
}

\section{Estrategias de comunicación en la mejora de los factores de riesgo cardiovascular y enfermedades cardiovasculares en el Proyecto Karelia del Norte}

\author{
Noël C. Barengo \\ (Mar del Plata, Argentina) \\ Fundación para la Prevención y Control de las Enfermedades Crónicas No Transmisibles en \\ América Latina (FunPRECAL) \\ Jaakko O. Tuomilehto \\ (Helsinki, Finlandia) \\ HJELT Institute, University of Helsinki \\ Aulikki Nissinen y Pekka Puska \\ (Helsinki, Finlandia) \\ National Institute for Health and Welfare
}

\begin{abstract}
Palabras clave
comunicación

innovación

líderes de opinión

información

riesgo cardiovascular

$\underline{\underline{\text { Resumen }}}$

A partir de 1972 se llevó a cabo un programa comunitario integral para el control de las enfermedades cardiovasculares (ECV) en el norte de Karelia, Finlandia. El objetivo central del programa era reducir la prevalencia de fumadores, la concentración de colesterol y los valores de presión arterial en la población de la provincia. El Proyecto Karelia del Norte ilustra los objetivos fundamentales de promoción de la salud, comunicación para la salud y la difusión de las innovaciones con los líderes de opinión. Las actividades específicas del proyecto sirven como ejemplos de cómo los conceptos de las ciencias sociales y del comportamiento se pueden aplicar para conseguir en las comunidades una disminución de los factores del riesgo de las enfermedades crónicas no transmisibles, con el objetivo de reducir la carga de dichas enfermedades. El enfoque basado en la reorganización de la comunidad y las estrategias de comunicación para la salud representan una innovación en el control de las enfermedades crónicas. La reorganización de la comunidad implica la participación de diversos sectores de la sociedad, incluyendo a los medios de comunicación. Las experiencias de Karelia del Norte destacan el importante papel desempeñado por los líderes de opinión, los medios de comunicación locales y las estrategias de comunicación para la salud. La buena comprensión de la comunidad y una estrecha colaboración con una serie de organizaciones ha sido un elemento esencial del éxito. Este artículo explica las estrategias utilizadas en las campañas en lo que se refiere (1) a la información para educar a la gente sobre su salud y (2) a la persuasión para motivar a la gente a adoptar medidas saludables.
\end{abstract}




\section{Communication strategies in improving cardiovascular risk factors and cardiovascular diseases in the North Karelia Project}

\author{
Keywords \\ communication \\ innovation \\ opinion leaders \\ information \\ cardiovascular risk
}

\begin{abstract}
Between 1972 and 1982, a comprehensive community program to control cardiovascular diseases (CVD) was carried out in North Karelia, Finland. The program's main objective was to reduce the prevalence of smoking, the concentration of cholesterol, and raised blood-pressure among the area's population. The North Karelia Project illustrates the fundamental goals of health promotion, health communication and sharing of innovations using opinion leaders. The project's specific activities serve as examples of how concepts from social and behavioral sciences can be applied so as to reduce risk factors of transmissible chronic diseases in communities in order to reduce the burden of said diseases. The project's approach is based on reorganizing the community and its health communication strategies represent an innovation in chronic disease control. Reorganizing the community entails the participation of various social groups such as opinion leaders and the media. The experiences in North Karelia emphasize the important role played by opinion leaders, local media, and health communication strategies. An accurate understanding of the community and close collaboration with a series of organizations have been essential elements for success. This article explains the strategies used in campaigns regarding (1) information to educate people about their health and (2) persuasion to motivate people to take healthy measures.
\end{abstract}

\section{Cómo citar el artículo}

Barengo, N. C.; Tuomilehto, J. O.; Nissinen, A. y Puska, P. (2011). Estrategias de comunicación en la mejora de los factores de riesgo cardiovascular y enfermedades cardiovasculares en el Proyecto Karelia del Norte. Revista de Comunicación y Salud, 1(1), pp. 45-54.

DOI: http://doi.org/10.35669/revistadecomunicacionysalud.2011.1(1).45-54

\section{Introducción}

Después de la Segunda Guerra Mundial, las enfermedades crónicas, en particular las enfermedades cardiovasculares, se convirtieron en un importante problema de salud pública en los países industrializados. Estas enfermedades se consideraban como enfermedades de la opulencia. Finlandia tuvo la mayor tasa mundial de muertes por enfermedades cardiovasculares en la década de 1960 (Puska, 1989: 169-173). Los hombres de entre 35 y 64 años (edad de trabajo) estaban muriendo en gran número. Los peores índices se daban en el este del país, con las cifras más altas en la provincia de Karelia del Norte (Keys, 1980).

En 1971, representantes de la provincia de Karelia del Norte firmaron una petición a las autoridades nacionales solicitando ayuda urgente para reducir la carga de enfermedades cardio- 
vasculares en la provincia. En respuesta a la petición y en un intento por evitar un aumento de la incidencia de las enfermedades cardiovasculares, las autoridades finlandesas, con el apoyo de expertos y la ayuda de la Organización Mundial de la Salud, pusieron en marcha el Proyecto Karelia del Norte en 1972 (Puska, 2008: 26-29; Puska, 1999: 9-13; Puska, 1981). Además, eligieron la provincia de Kuopio como región de control, para comparar si las actividades en Karelia del Norte reflejaban una diferencia en los hábitos de vida y factores del riesgo entre las dos provincias.

El marco teórico del proyecto incorporó el cambio de comportamiento, las estrategias de la comunicación de salud y una reorganización comunitaria. Las intervenciones incluyeron campañas en los medios de comunicación, la colaboración con la industria alimentaria, la participación de los servicios de salud locales y de organizaciones comunitarias, y reformas agrícolas (Puska, 1995; Nissinen, 1988: 49-56).

El objetivo del proyecto era cambiar el estilo de vida relacionado con el riesgo de las enfermedades cardiovasculares en la provincia a través de acciones comunitarias dirigidas a la prevención e información, así como trabajar con una red de organizaciones, incluyendo a las ONG, el sector privado y los políticos responsables. La idea general era transformar el entorno social y físico de Karelia del Norte. La mayoría de los subprogramas que se llevaron a cabo consiguieron los objetivos fundamentales de promoción de la salud e ilustraron los principios teóricos en acción.

El reconocimiento del papel desempeñado por los factores de riesgo relacionados con el estilo de vida en el aumento de las tasas de enfermedad fue clave para la elección de la estrategia principal del proyecto: un enfoque basado en la comunidad, en la que las intervenciones preventivas fueron dirigidas no solo a las personas que viven con una enfermedad crónica, o incluso aquellos con alto riesgo, sino a toda la población.

Dentro de la literatura sobre la promoción de la salud cardiovascular comunitaria y la prevención de enfermedades crónicas, el proyecto de Karelia del Norte es considerado como un programa modelo, que logró importantes reducciones en los factores de riesgo y la mortalidad asociados a enfermedades cardiovasculares (Blashko, 1990: 49-59; Brownson, 1997: 333345; Burgess, 2000: 30-38; Pratt, 1999: 81-90). Esta opinión está confirmada por las estadísticas a largo plazo en la mejora de las tasas de mortalidad por causas específicas durante y después de la intervención. Esta intervención de la comunidad ha acreditado el logro de una reducción del 73\% en la tasa de mortalidad cardiovascular entre 1969 y 1995 (Puska, 1995: 63-66; Laatikainen, 2005: 764-773).

El objetivo de este trabajo es explicar las estrategias y los métodos utilizados en la educación para la salud, la comunicación para la salud y la motivación de la gente para cambiar el comportamiento y vivir de forma más saludable.

\section{Las estrategias de intervención en materia de comunicación}

El proyecto de Karelia del Norte se basó en varios modelos generales utilizados en los progra- 
mas de promoción de la salud en ese momento (Kirscht, 1974: 387-408; Rosenstock, 1974: 328-335). El programa completo se integró en los servicios sociales y de salud de la provincia (Puska, 1985: 147-193). Se usaron los cinco pasos esenciales para impulsar el cambio de comportamiento de la gente (McAlister, 1982: 43-50):

1. Mejora de los servicios de prevención, para identificar a las personas de alto riesgo de padecer la enfermedad y proporcionar atención médica adecuada.

2. Información, para educar a la gente acerca de su salud y cómo se puede mantener.

3. Persuasión, para motivar a la gente a adoptar medidas saludables.

4. Formación, para aumentar las habilidades de autocontrol, la gestión ambiental, y la acción social, la organización de la comunidad para crear un apoyo social y el poder para la acción social.

5. Cambio ambiental, para crear oportunidades para las acciones de salud y mejorar una serie de condiciones desfavorables.

En este artículo se explican con más detalle los pasos dos y tres, referidos a la información y la persuasión.

\subsection{Información}

\subsubsection{Campañas de información}

La cooperación con cualquier programa o servicio diseñado para prevenir la enfermedad depende del grado en que la comunidad esté informada sobre los efectos y la importancia del programa (McAlister, 1982: 43-50). Así, una de los principales objetivos de la promoción de la salud es educar a la gente acerca de su salud y cómo se puede mantener. Ejemplos de este tipo de actividad son informar al público que las enfermedades cardiovasculares se pueden prevenir con medidas adecuadas, y explicar el propósito y la naturaleza de estas medidas. Sin embargo, no siempre es fácil comunicar adecuadamente las ideas nuevas y complejas en una gran población que está sujeta a la información de muchas fuentes (McAllister, 1982: 43-50).

El diseño de campañas eficaces de información puede ser facilitado por la aplicación de principios prácticos derivados de la investigación y la teoría de la comunicación. Por ejemplo, la investigación muestra que los medios de comunicación, especialmente a través de las noticias, influyen poderosamente en lo que la gente habla y piensa, y cómo juzga la importancia de los diversos problemas sociales (Kirscht, 1974: 387-408; Rosenstock, 1974: 328-335). La teoría sugiere que las nuevas ideas deben atravesar varios pasos de la comunicación interpersonal para llegar a la población general (Kirscht, 1974: 387-408). Los mensajes deben ser simples y repetidos con frecuencia si se quiere ser comprendido y retenido (Rosenstock, 1974: 328335).

El Proyecto Karelia del Norte ofreció varias ilustraciones de la aplicación de estos principios. 
El personal del proyecto fue capaz de atraer la atención intensa y frecuente de los medios de comunicación en la región, especialmente de los periódicos y la radio. Entre 1972 y 1977, se publicaron en los periódicos locales un total de 1.509 artículos relacionados con factores de riesgo cardiovascular, su gestión y las actividades del programa (Puska, 1985: 147-193). Esto fue tres veces más que en el área de control, la provincia de Kuopio. Durante ese mismo período fueron distribuidos más de un millón y medio de boletines, folletos, pósters, carteles, pegatinas y otros materiales educativos. Para estimular aún más la comunicación interpersonal, se les pidió a muchos grupos y organizaciones que distribuyeran materiales en su trabajo diario o que cooperaran en la organización de reuniones de educación sanitaria. Se llevaron a cabo un total de 251 reuniones, llegando a más de 20.000 miembros de la comunidad. Los grupos comunitarios y organizaciones que participaron en estas actividades incluyeron lugares de trabajo, escuelas, tiendas y comercios, clubes y organizaciones voluntarias. La información sobre el programa y sus objetivos se difundió rápidamente (Puska, 1981).

En un período de cinco años, el conocimiento de los factores de riesgo de enfermedad cardiovascular mejoró tanto en Karelia del Norte como en la zona de control, según las encuestas poblacionales (Puska, 1979: 1.173-1.178). Hubo diferencias marcadas del conocimiento en la población de Karelia del Norte a los 10 años del proyecto. La población general de Karelia del Norte mostró un aumento de entre el 10 y el 15 por ciento en la proporción de respuestas correctas a preguntas de la encuesta diseñada para medir el conocimiento, la conciencia y la comprensión de factores de riesgo cardiovascular. Sin embargo, no hubo diferencias significativas en los cambios en Karelia del Norte y en la zona de control, probablemente debido a la creciente atención de los medios de comunicación nacional que sirven a ambas provincias.

\subsubsection{Comunicar nuevas ideas}

En la comunicación de ideas nuevas el personal del proyecto buscó deliberadamente una mezcla de fuentes diferentes para la diseminación de los mensajes y para alcanzar la máxima credibilidad de la información distribuida entre la población (Neittaanmaki, 1980: 1-7; Puska 1986: 437-446). Consejos y apoyo fueron obtenidos de instituciones tan prestigiosas como las ONG. La población de Karelia del Norte fue objeto de una comunicación persuasiva especialmente intensa que le llegaba de respetados expertos, médicos y otros agentes sociales, a quienes se animó a difundir y apoyar las nuevas ideas en la comunidad.

Los médicos y enfermeras de salud pública fueron una parte importante del sistema de comunicación (McAlister, 1982: 43-50). Las encuestas mostraron que durante un período de cinco años, en Karelia del Norte estos dos grupos profesionales distribuyeron más información y estuvieron mucho más involucrados en los contactos activos con la toma de decisiones de las organizaciones comunitarias que en el área de control: sólo el ocho por ciento de las decisiones había sido explícitamente asesorada por una enfermera de salud pública para cambiar los hábitos alimenticios en el área de control, frente a más del 20 por ciento en Karelia del Norte (Puska, 1981).

El contenido de los mensajes fue construido cuidadosamente para prevenir y suprimir los contra-argumentos. Debido a que muchos pobladores locales se dedicaban a ocupaciones activas, 
estaban firmemente convencidos de que una dieta alta en grasa de carne era necesaria para las personas trabajadoras. Los mensajes estuvieron dirigidos a disminuir la ingesta de grasas, con argumentos como que había leñadores vegetarianos y que uno de los más famosos corredores de distancia de Finlandia, H. Kolemainen, era vegetariano. Se mencionó en repetidas ocasiones al hecho de que la dieta recomendada, baja en grasa, era más "tradicional" en Karelia del Norte que la dieta actual, rica en grasas. Las comparaciones de los cambios en los hábitos alimenticios reveló que la reducción en el consumo reportado de grasa en Karelia del Norte fue significativamente mayor que la observada en la zona de control (Puska, 1981).

2.1.3. El uso de líderes de opinión para promover la difusión de las innovaciones de la salud en una comunidad

El enfoque de la teoría de la difusión de una innovación se centra en la difusión de un estilo de vida más saludable para la reducción de riesgos de enfermedades crónicas (innovación), que se difunden en el transcurso del tiempo a través de las redes comunitarias para llegar a toda la población. Esta difusión conduce al cambio social. Mientras que los medios de comunicación son eficaces para difundir innovaciones, los contactos interpersonales son más eficaces en cambiar las actitudes que condicionan los hábitos de vida. El proceso de difusión de la innovación se basa en el conocimiento, la persuasión, la decisión y la confirmación. Las personas pueden ser clasificadas como innovadores, adoptadores tempranos, mayoría temprana o tardía, y rezagados. La estructura social de la comunidad influye en la velocidad de difusión. Estos principios básicos desarrollados por Rogers $(1971,1983)$ encajan con el flujo de dos pasos de las nuevas ideas a través de los líderes de opinión (Katz y Lazarsfeld, 1955).

En primer lugar, las nuevas ideas vienen de los medios de comunicación, y luego son transmitidas por los líderes de opinión después de algunas modificaciones. Entonces, la mayoría de las personas están influidas por los contactos interpersonales con estos líderes, que promueven o bloquean el proceso de innovación-difusión. Los líderes de opinión son quienes están más expuestos a los medios de comunicación y a los contactos sociales, tienen la condición social más alta y más influencia como agente de cambio, y suelen mostrar más capacidad de innovación (Puska, 1986: 437-446). Además, a menudo se encuentran en una posición clave para influir en una mayor adopción de la innovación si están satisfechos con ella y se comprometen a comunicar esta opinión a través de redes interpersonales (Rogers, 1983).

En el proyecto de Karelia del Norte, el uso sistemático de líderes de opinión se inició en 1975 para facilitar el cambio de comportamiento. En total, más de 800 líderes de opinión fueron capacitados en un total de 44 reuniones para promover la reducción de los factores de riesgo de enfermedad cardiaca (Puska, 1986: 437-446). Los líderes de opinión fueron entrenados en técnicas sencillas para el refuerzo verbal y el fomento de dejar de fumar, cambios en la dieta y otros comportamientos preventivos. Ellos también fueron capacitados para detectar problemas de la salud cardiovascular en tiendas, escuelas, oficinas y lugares de encuentro, y para persuadir a las personas para que lleven a cabo los cambios necesarios (Puska, 1986: 437446). Por lo tanto, la actividad principal de los líderes de opinión era a través de la observación y discusión con la gente de las principales cuestiones sobre salud. Los debates acerca de sus propias experiencias, consejos prácticos y la remisión al personal del proyecto de Karelia del 
Norte fueron instrumentos potencialmente poderosos de la persuasión.

\subsubsection{Cambio del comportamiento}

El Proyecto Karelia del Norte tuvo mucho cuidado con el concepto de "alto riesgo". Desde el comienzo se tuvo claro que todos los mensajes que provocan miedo deben ir acompañados de recomendaciones claras y alcanzables para reducir el miedo (McAlister, 1982: 43-50). A las personas con presión arterial elevada o colesterol se les dijo que su condición era potencialmente grave, pero que mediante medidas simples se podría aliviar el problema. No se hizo distinción entre niveles de factores de riesgo "patológicos" y "seguros". Las enfermeras de salud pública y los educadores señalaron continuamente que los factores de riesgo en la población eran altos y que todo el mundo tenía razones para hacer cambios. Las encuestas mostraron que los cambios de comportamiento fueron similares entre los grupos de población con variados niveles de riesgo inicial, y que no había tendencia a una mayor ansiedad o trastornos psicosomáticos en aquellos con factores de riesgo excepcionalmente elevados.

Uno de los enfoques utilizados en el marco teórico fue el del uso de las emociones para la persuasión, que se basa en buscar una asociación emocional en lugar de argumentos racionales. Hubo un esfuerzo consciente por parte del proyecto para que la población asociara los objetivos del proyecto con la identidad y el orgullo de la provincia. Se instó a la gente a participar y hacer cambios, no sólo para sí mismos sino para Karelia del Norte. Por ejemplo, carteles con el mensaje "no fume aquí, estamos en el Proyecto Karelia del Norte" estaban por todas partes y fomentado una especie de patriotismo local (Puska, 1995; McAlister, 1982: 43-50).

Otro enfoque utilizado fue el conductual, que tampoco se basa en argumentos racionales. El personal del proyecto conoció a cientos de actores locales influyentes, con quienes discutieron los diversos problemas de la comunidad. A casi todo el mundo se le pidió hacer al menos algún tipo de compromiso menor de comportamiento a favor del proyecto; por ejemplo, miles de ciudadanos colaboraron con pequeñas acciones como la exhibición de pegatinas y carteles. Estas acciones, sin duda, influyeron para que los individuos tuvieran una visión más favorable del proyecto y sus recomendaciones.

2.1.5. Campañas de televisión como herramienta de comunicación en la promoción de la salud en el Proyecto Karelia del Norte

El poder de la televisión en la promoción de la salud se demostró a partir de 1978 con el programa Claves para la salud, coproducido por el Instituto Nacional de Salud Pública y por el canal de televisión Finlandia 2 (Puska, 1987: 263-269). Se emitió una serie de cinco programas que contó con un grupo de voluntarios que tenían un alto riesgo de enfermedad debido a sus hábitos poco saludables y con dos educadores de salud, quienes daban consejos a los voluntarios y los espectadores para hacer cambios en los comportamientos de salud.

Los programas utilizaron el mismo formato. Los voluntarios o grupo de estudio consistió en 6 a 10 personas de la comunidad que trataban de modificar sus hábitos relacionados con la salud, siguiendo las instrucciones de los dos expertos del proyecto. Los participantes fueron 
personas de diferentes niveles socioeconómicos, que tenían algunos estilos de vida poco saludables y se ofrecían voluntariamente para servir como modelos en sus intentos de cambiar las conductas de salud. Los miembros de estos grupos por lo general eran fumadores y con frecuencia tenían una dieta alta en grasas. Algunos de los participantes presentaban sobrepeso, además del colesterol y niveles de presión arterial elevados. Los participantes trataron de reducir sus factores de riesgo y compartieron sus experiencias en una serie de programas de televisión, emitidos con intervalos de 1 a 2 semanas. Las sesiones fueron grabadas en el estudio de televisión y transmitidas más tarde con muy pocas correcciones con el fin de comunicar el mensaje con la mayor naturalidad posible a los espectadores.

Durante las sesiones, los participantes recibieron asesoramiento de los expertos del proyecto acerca de cómo cambiar gradualmente sus hábitos de salud. Las emisiones también incluyeron ejemplos de los problemas prácticos con los que podían enfrentarse a la hora de hacer los cambios necesarios. Al incluir tanto los problemas a los que se enfrentaban los participantes como las estrategias para superarlos, el programa de TV ayudó a que los espectadores se identificaran mejor con ellos y a comprender los cambios promovidos en los hábitos de salud.

El impacto del programa televisivo fue evaluado en la última de las cinco series. Los resultados de las encuestas de evaluación indicaron que los índices de audiencia eran altos. En todo el país, el 27 por ciento de los hombres y el 35 por ciento de las mujeres informaron que habían visto al menos tres partes de la serie. Los cambios registrados en los comportamientos eran sustanciales entre los espectadores que habían visto varias partes de la serie. Los intentos para dejar de fumar se duplicaban entre los espectadores del programa en comparación con el total de fumadores ( 7,1 frente al 3,6 por ciento). Las reducciones reportadas en el consumo de grasas fueron del 27,2 por ciento para los hombres y del 15,0 por ciento para las mujeres. Los cambios reportados en el área de demostración de Karelia del Norte eran más altos que en el resto del país, principalmente debido a mayores tasas de visualización (Puska, 1987: 263-269).

\section{Conclusiones}

El Proyecto Karelia del Norte fue el primer programa integral basado en la comunidad para demostrar un impacto positivo en la mortalidad cardiovascular y la discapacidad, utilizando fundamentalmente estrategias de promoción de la salud, comunicación para la salud y la difusión de las innovaciones en la comunidad con los líderes de opinión. Las actividades específicas del proyecto sirven como ejemplo de cómo los conceptos de las ciencias sociales y del comportamiento se pueden aplicar para conseguir una reducción en la gravedad de los factores del riesgo de las enfermedades cardiovasculares en las comunidades, con el objetivo de reducir la carga de dichas enfermedades. Desde sus inicios, el proyecto recibió un gran interés internacional, en particular por la metodología utilizada, las actividades realizadas y las experiencias acumuladas.

Durante los más de de 30 años transcurridos desde la puesta en marcha del Proyecto Karelia del Norte, se han producido cambios muy grandes en toda la población en los hábitos de vida y los factores de riesgo de las enfermedades cardiovasculares (Puska, 2008: 26-29). Por ejemplo, se ha registrado un cambio drástico en los hábitos alimenticios de la población. Al comienzo 
del proyecto, casi todas las personas utilizaban la mantequilla en el pan y en la cocina; en la actualidad, menos del $5 \%$ de la gente utiliza la mantequilla en el pan y cerca de $60 \%$ de los hogares utiliza principalmente aceite vegetal para cocinar. En la década de 1970, más de la mitad de los hombres fumaba y ahora sólo el $20 \%$ de los hombres fuma todos los días. El nivel promedio del colesterol de la población en Karelia del Norte se ha reducido más del $20 \%$.

El impacto de estos cambios en la salud pública ha sido espectacular. Según las últimas estadísticas, la tasa anual de mortalidad estandarizada por edad para la enfermedad cardiaca entre los hombres en edad de trabajar en Karelia del Norte fue en 2006 un $85 \%$ menor que la registrada durante el período 1969-1971; a nivel nacional, la reducción fue de alrededor del 80\%, entre otras cosas porque las estrategias y acciones que demostraron ser exitosas en Karelia del Norte luego fueron reproducidas en el resto del país. Además, las tasas de ictus y de cánceres en los hombres se redujeron el $48 \%$ y $44 \%$, respectivamente, entre 1972 y 1992 . Estos cambios han provocado una reducción de la mortalidad por todas las causas entre las personas en edad de trabajar de más del $50 \%$. La tercera edad y los grupos de mayores se han beneficiado de este efecto preventivo, y la tasa de mortalidad de enfermedades cardiovasculares se redujo el $47 \%$ en hombres y el $52 \%$ en las mujeres. En consonancia con esta mejora en el perfil de la enfermedad, la salud subjetiva y el bienestar de la población también han mejorado.

El Proyecto Karelia del Norte representó una innovación en el control de las enfermedades crónicas. La reorganización de la comunidad implicó la participación de diversos sectores de la sociedad, incluyendo a los medios de comunicación. Las experiencias de Karelia del Norte destacan el importante papel desempeñado por los líderes de opinión, los medios de comunicación de ámbito local y la comunicación para la salud. Una buena comprensión de la comunidad y la estrecha colaboración con sus organizaciones han sido dos elementos esenciales del éxito.

\section{Referencias}

Blashko, Carl y Paterson, John (1990). You and your heart. Promoting preventive health education through the mass media. International Journal for the Advancement of Counselling. Vol. 13, pp. 49-59.

Brownson R.C.; Mayer J.P.; Dusseault P.M. et al. (1997). Developing and evaluating a cardiovascular risk reduction project. American Journal of Health Behavior. Vol. 21, pp. 333-345.

Burgess, Record N.; Harris D.E.; Record, S.S. et al. (2000). Mortality impact of an integrated community cardiovascular health program. American Journal of Preventive Medicine. Vol. 19, pp. 30-38.

Katz, Elihu y Lazarsfeld, Paul (1955). Personal influence: the part played by people in the flow of mass communications. New York: Free Press.

Keys, Ancel (1980). Seven countries. A multivariate analysis of death and coronary heart disease. Cambridge: Harvard University Press.

Kirscht, J.P. (1974). The health belief model and illness behavior. Health Education Monographs. Vol. 2, pp. 387-408.

Laatikainen, T.; Critchley, J.; Vartiainen, E., et al. (2005). Explaining the decline in coronary 
heart disease mortality in Finland between 1982 and 1997. American Journal of Epidemiology. Vol. 162; pp. 764-773.

McAlister, A. et al. (1982). Theory and action for health promotion. Illustrations from the North Karelia Project. American Journal of Public Health. Vol. 72, pp. 43-50.

Neittaanmaki, L.; Koskela, K.; Puska, P. y McAlister, A. (1980). The role of lay workers in a community health education in the North Karelia Project. Scandinavian Journal of Social Medicine. Vol. 8, pp. 1-7.

Nissinen, Aulikki; Tuomilehto, Jaakko et al. (1988). From pilot project to national implementation: experiences from the North Karelia Project. Scandinavian Journal of Primary Health Care. Suppl. 1, pp. 49-56.

Pratt, C.A.; Hurst, R.; Williams, K.P. et al. (1999). Evaluating cardiovascular disease prevention programs in African American communities. Journal of Public Health Management and Practice. Vol. 5, pp. 81-90.

Puska, Pekka; Tuomilehto, Jaakko et al. (1979). Changes in coronary risk factors during comprehensive five-year community programme to control cardiovascular diseases (North Karelia Project). British Medical Journal. Vol. 2, nº 6.199, pp. 1173-1178.

Puska, P.; Tuomilehto, J.; Salonen, J. et al. (1981). The North Karelia Project: Evaluation of a comprehensive community program for control of cardiovascular diseases in 1972-1977 in North Karelia, Finland. Monográfico. Copenhage: WHO/EURO.

Puska, Pekka; Nissinen, Aulikki et al. (1985). The Community-Based Strategy to Prevent Coronary Heart Disease: Conclusions from the Ten Years of the North Karelia Project. Annual Review of Public Health. Vol. 6, pp. 147-193.

Puska, Pekka; Tuomilehto, Jaakko et al. (1989). The North Karelia Project: 15 Years of Community-based Prevention of Coronary Heart Disease. Annals of Medicine. Vol. 21, pp. 169-173.

Puska, Pekka; Koskela, Kaj et al. (1986). Use of lay opinion leaders to promote diffusion of health innovations in a community programme: lessons learned from the North Karelia Project. Bulletin of the World Health Organization. Vol. 64, n 3, pp. 437-46.

Puska, Pekka (1995). Communication with the population: the North Karelia Project experience. Journal of Human Hypertension. Vol. 9, n 1, pp. 63-6.

Puska, Pekka; Tuomilehto, Jaakko; Nissinen Aulikki y Vartiainen Erkki. (1995). The North Karelia Project: 20-year results and experiences. Helsinki: National Public Health Institute.

Puska, Pekka (1999). The North Karelia Project: from community intervention to national activity in lowering cholesterol levels and CHD risk. European Heart Journal. Vol. 1, Suppl., pp. 9-13.

Puska, Pekka (2008). The North Karelia Project: 30 years successfully preventing chronic diseases. Diabetes Voice. Vol. 53, pp. 26-29.

Rogers, Everett y Shoemaker, Floyd (1971).Communication of innovations. A cross-cultural approach. New York: Free Press.

Rogers, Everett (1983). Diffusion of innovations. New York: Free Press.

Rosenstock, I.M. (1974). Historical origins of the health belief model. Health Education Monographs. Vol. 2, pp. 328-35. 